men ausgeschlossen, die Patienten also weniger krank waren als etwa in der STAR*D-Studie [1] und dem Berliner Stufenplan [2], wo die Remissionsraten nicht so hoch waren. Und auch die gewählte Medikation stellt vielleicht nicht die Behandlung erster Wahl hierzulande dar. Dennoch spricht einiges dafür, sich bei der Behandlung psychischer Erkrankungen mehr an objektivierbaren Skalen zu orientieren. So zeigte sich in dieser Studie, dass die Aufdosierung der Medikation in der an Skalen orientierten Gruppe schneller erfolgte und Dosisanpassungen häufiger waren, was auch eine Ursache für die sehr guten Response- und Remissionsraten sein könnte. Trotz der oben genannten Einschränkungen scheint es wichtig und sinnvoll, diese Daten zu replizieren, denn sie stellen einen Ansporn dar, das eigene klinische Handeln immer wieder auf Objektivität und Leitlinienkonformität zu überprüfen und damit effektiver zu machen. Dazu liefert die Studie einen wichtigen Diskussionsbeitrag.
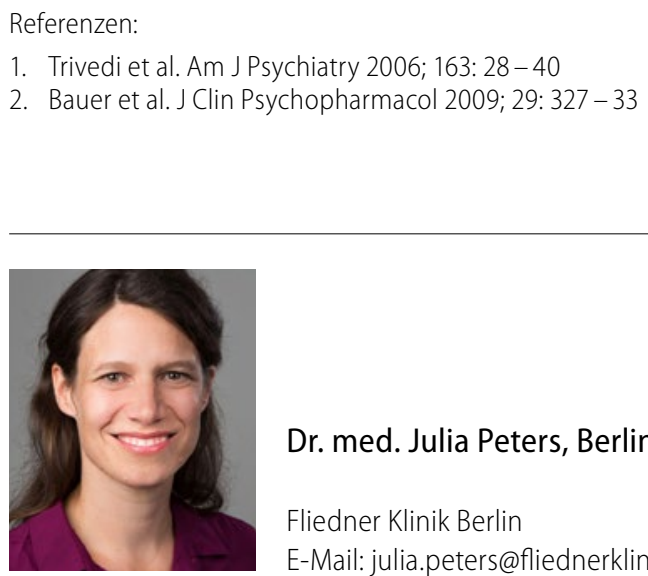

Dr. med. Julia Peters, Berlin

Fliedner Klinik Berlin

E-Mail: julia.peters@fliednerklinikberlin.de

\title{
Effektive Schlaganfallprävention
}

Fragestellung: Ist Apixaban zur Schlaganfallprävention bei $\mathrm{Pa}$ tienten mit Vorhofflimmern sowie leichten und mittelgradigen Herzklappenschäden zur Schlaganfallprävention wirksam?

Hintergrund: Patienten mit Vorhofflimmern haben ein erhöhtes Schlaganfallrisiko. Es ist besonders hoch, wenn zusätzlich eine rheumatische Herzerkrankung mit Herzklappendefekten vorliegt. Für diese Patienten sind die neuen Antikoagulanzien nicht geeignet.

Avezum A, Lopes RD, Schulte PJ et al. Apixaban in comparison with warfarin in patients with atrial fibrillation and valvular heart disease: Findings from the Apixaban for Reduction in Stroke and Other Thromboembolic Events in Atrial Fibrillation (ARISTOTLE) trial. Circulation 2015; 132: $624-32$
Patienten und Methodik: Die ARISTOTLE-Studie verglich bei Patienten mit Vorhofflimmern das neue Antikoagulans (NOAK) Apixaban, einen Faktor-Xa-Hemmer, in einer Dosis von $2 \mathrm{x}$ 2,5 mg mit INR-angepasstem Warfarin [1]. In die Studie konnten auch Patienten mit
Aorten-Stenosen, leichten Mitralklappenstenosen, Trikuspidalstenosen und biologischen Herzklappen eingeschlossen werden. Primärer Endpunkt war die Häufigkeit von Schlaganfällen und systemischen Embolien.

Ergebnisse: Von den 18.200 Studienteilnehmern wiesen 4.808 $(26,4 \%)$ Veränderungen an den Herzklappen auf oder hatten biologische Herzklappen. Im Vergleich zu der übrigen Studienpopulation hatten diese Patienten ein höheres Risiko für Schlaganfälle und Blutungskomplikationen. Das Schlaganfall- und Herzinfarktrisiko war wie in der Gesamtstudie für Apixaban um $30 \%$ reduziert. Schwerwiegende Blutungen waren um $20 \%$ reduziert. Die Mortalität war nicht verändert.

Schlussfolgerungen: In der ARISTOTLE-Studie hatte ein Viertel aller Patienten leichte oder mittelschwere Herzklappenerkrankungen. Bei diesen Patienten war Apixaban überlegen im Vergleich zu Warfarin für die Schlaganfallprävention und führte $\mathrm{zu}$ weniger Blutungskomplikationen. Das ist mit den Ergebnissen der Gesamtstudie vergleichbar.

\section{- Kommentar von Hans-Christoph Diener, Essen}

\section{Auch diese Patienten profitieren von Apixaban}

In den meisten Studien, welche die neuen Antikoagulanzien mit Vitamin-K-Antagonisten verglichen, waren Patienten mit Herzklappenerkrankungen ausgeschlossen. Dies war für rheumatische Herzerkrankungen und hochgradige Mitralklappenstenosen auch in der ARISTOTLE-Studie der Fall. Patienten mit leichten Stenosen und Stenosen an den Herzklappen sowie Patienten mit biologischen Herzklappen konnten eingeschlossen werden. Sie machten etwa ein Viertel der Studienpopulation aus. Es zeigte sich beruhigender Weise, dass für diese $\mathrm{Pa}$ - tienten der Nutzen von Apixaban im Vergleich zu Warfarin sowohl für die Reduktion von Schlaganfällen als auch für die Reduktion von schwerwiegenden Blutungen bestehen bleibt. Deshalb kann diese Population problemlos mit Apixaban behandelt werden.

\footnotetext{
Referenz:

1. Granger CB et al. N Engl J Med 2011; 365: 981 - 92
} 